\title{
Comparison of the Efficacies of Debris Removal With Four Different Irrigation Techniques
}

\author{
Emre Iriboz ${ }^{\mathrm{a}, \mathrm{c}}$, Koral Bayraktar ${ }^{\mathrm{a}}$, Dilek Turkaydin ${ }^{\mathrm{a}}$, Bilge Tarcin ${ }^{\mathrm{b}}$, Hesna Sazak Ovecoglu ${ }^{\mathrm{a}}$
}

\begin{abstract}
Background: In this study, we compared the effectiveness of debris removal among the EndoVac (EV), passive ultrasonic irrigation (PUI), self-adjusting file (SAF) and needle irrigation (NI) techniques.
\end{abstract}

Methods: Forty-two single-canal teeth were divided into four groups of 10 teeth each and two controls. The experimental groups were NI, SAF, PUI and EV. After irrigation protocols, the teeth were sectioned at 1 and $3 \mathrm{~mm}$ from the apex using a precision saw. The specimens were observed under stereomicroscope at $\times 128$ magnification and digitally photographed. The amount of remaining debris was calculated as a percentage of the canal lumen area.

Results: The amounts of debris remaining at $1 \mathrm{~mm}$ in NI, SAF, PUI and EV groups were $18.11 \%, 7.12 \%, 12.71 \%$ and $4.42 \%$, respectively. The amounts of debris at $3 \mathrm{~mm}$ in NI, SAF, PUI and EV groups were $9.61 \%, 5.22 \%, 8.42 \%$ and $2.61 \%$ respectively. The amount of debris in the EV group was significantly lower than the other groups $(\mathrm{P}<0.01)$. The amount of debris at $3 \mathrm{~mm}$ was significantly lower than at $1 \mathrm{~mm}(\mathrm{P}<0.01)$.

Conclusion: EV irrigation resulted in significantly less debris at both 1 and $3 \mathrm{~mm}$ from the apex compared with the other irrigation techniques.

Keywords: Debris removal; EndoVac; Irrigation; Passive ultrasonic irrigation; SAF

Manuscript accepted for publication July 30, 2014

${ }^{a}$ Department of Endodontics, Faculty of Dentistry, Marmara University, Buyukciftlik St. No. 6 Nisantasi, Istanbul, Turkey

${ }^{b}$ Department of Restorative Dentistry, Faculty of Dentistry, Marmara University, Buyukciftlik St. No. 6 Nisantasi, Istanbul, Turkey

${ }^{\mathrm{c}}$ Corresponding Author: Emre Iriboz, Department of Endodontics,

Faculty of Dentistry, Marmara University, Buyukciftlik St. No. 6

Nisantasi, Istanbul, Turkey. Email: eriboz@yahoo.com

doi: http://dx.doi.org/10.14740/jcs242e

\section{Introduction}

Thorough debridement is crucial for the long-term success of root canal treatment. Hand and rotary instrumentation techniques produce an irregular, granular and amorphous layer covering the root canal dentin, referred to as the smear layer. The presence of a smear layer was shown to negatively influence the coronal and apical seal of root canal-treated teeth [1]. Removal of the smear layer is recommended because it results in a more thorough disinfection of the root canals and ensures better adaptation between the obturating materials and the canal walls.

Hand and/or rotary instrumentation along with needle irrigation (NI) does not effectively debride the entire root canal $[2,3]$. Additionally, the intricacies within the apical third of the root canal render complete debridement a clinical challenge [4]. Irrigation is an essential part of the root canal debridement because cleaning beyond what can be achieved by root canal instrumentation alone is possible [5]. The mechanical and chemical effectiveness of the irrigation delivery system depends on its ability to deliver irrigants to the apical third and non-instrumented regions of the canal space. Additionally, the system should create a strong current to carry the debris away from the canal walls [6].

Irrigation solutions are often delivered with a 30- or 27-G endodontic slot-tipped needle placed into the canal just short of the apex. The depth of needle penetration is dependent on the size and morphology of the canal, rendering this technique difficult [7].

The self-adjusting file (SAF; ReDent Nova, Ra'anana, Israel) is hollow and designed as a thin cylindrical nickeltitanium lattice that adapts to the cross-section of the root canal. A single file is used throughout the procedure $[8,9]$. The resulting circumferential pressure allows the file's abrasive surface to gradually remove a thin, uniform hard-tissue layer from the entire root canal surface, resulting in a canal with a similar cross-section but with larger dimensions [10, 11]. This is also true for canals with an oval or flat crosssection, which are enlarged to a flat or oval cross-section of larger dimensions. The straightening of curved canals is also reduced; thus the original shape of the root canal is respected 
Table 1. Amounts of Remaining Debris in the Experimental Groups

\begin{tabular}{llllll}
\hline & NI & SAF & PUI & EV \\
\cline { 2 - 5 } Debris (\%) & Mean \pm SD & Mean \pm SD & Mean \pm SD & Mean \pm SD & P value \\
& $18.11 \pm 5.07$ & $7.12 \pm 1.59$ & $12.71 \pm 1.31$ & $4.42 \pm 0.71$ & $0.001^{* *}$ \\
$1 \mathrm{~mm}$ & $9.61 \pm 2.48$ & $5.22 \pm 1.37$ & $8.42 \pm 1.00$ & $2.61 \pm 0.46$ & $0.001^{* *}$ \\
$3 \mathrm{~mm}$ & $13.86 \pm 3.50$ & $6.17 \pm 1.38$ & $10.56 \pm 1.07$ & $3.51 \pm 0.45$ & $0.001^{* *}$ \\
\hline
\end{tabular}

One-way ANOVA test. ${ }^{* *} \mathrm{P}<0.01$. NI: needle irrigation; SAF: self-adjusting file; PUI: passive ultrasonic irrigation; EV: EndoVac.

both longitudinally and cross-sectionally [12].

Recently, with its gradually increasing popularity, passive ultrasonic activation of endodontic instruments has been suggested as a means to improve canal debridement [13], canal disinfection [14] and canal sealing [15]. Passive ultrasonic irrigation (PUI) has also been recommended for removing $\mathrm{Ca}(\mathrm{OH})_{2}$ from the root canal [16]. However, whether PUI as an effective irrigation method causes extrusion of irrigant from the apicalforamen remains unknown.

The EndoVac (EV; Discus Dental, Culver City, CA, USA) negative pressure irrigation system was developed to address the procedural challenge of delivering irrigants safely to the working length (WL). An EV placed to the WL resulted in significantly better debridement at $1 \mathrm{~mm}$ from the WL compared with NI, in teeth prepared to an ISO size \#36 or larger [17]. Water was used to compare the safety of the available irrigation systems; the results showed no extrusion in any of the EV samples [18]. An intracanal aspiration technique produced limited extrusion of the irrigant compared with conventional NI [19]. In this study, we compared apical extrusion of $\mathrm{NaOCl}$ delivered with a $27-\mathrm{G}$ irrigation needle, the EV, the SAF, or PUI during both instrumentation and the final irrigation of single-canal teeth. The effectiveness of debris removal using EV, PUI, SAF, or NI was compared.

\section{Materials and Methods}

Forty-two anterior teeth with a single root canal were used. All teeth were placed in physiological saline. The external surface of the teeth was cleaned with a hand scaler. The crown portion was removed using a diamond bur and the coronal part of the root was trimmed to $18 \mathrm{~mm}$ in length. The specimens were divided into four experimental groups according to the root canal irrigation system used: group 1 (n $=10), 27$-gauge NI; group $2(\mathrm{n}=10)$, SAF irrigation; group 3 $(n=10)$, PUI; group $4(n=10)$, the EV system. Teeth without irrigation were used as controls $(n=2)$.
The coronal portion was flared using \#2, \#3, and \#4 Gates Glidden (GG) drills (Mani Inc., Tochigi, Japan). The WL was determined by inserting a size- 15 file into the canal until the tip of the file was just visible at the apical foramen, and then subtracting $1 \mathrm{~mm}$ from that length. The teeth were mounted in resin blocks and the root canals were then instrumented to a master apical file size \#30 with ProTaper Universal rotary instruments (S1, S2, F1, F2 and F3; Dentsply). To maintain patency, recapitulation and $0.5 \mathrm{~mm}$ over insertion of a \#10 stainless steel hand file (DentsplyMaillefer) to the WL was performed after each instrumentation. After each file insertion, the root canal was irrigated with $1 \mathrm{~mL}$ of a freshly prepared $5.25 \% \mathrm{NaOCl}$ solution. The final irrigation was performed with $2 \mathrm{~mL}$ of $5.25 \% \mathrm{NaOCl}$, then $2 \mathrm{~mL}$ of $17 \%$ EDTA followed by another $2 \mathrm{~mL}$ of $5.25 \% \mathrm{NaOCl}$. Irrigation in the NI group was performed with a 27-G slottipped endodontic needle (Monoject, Tyco Healthcare, Mettawa, IL, USA) and syringe. The needle was placed short of the binding point or $2 \mathrm{~mm}$ from the WL and irrigants were delivered over $30 \mathrm{~s}$. In the SAF group, irrigants were delivered through the SAF according to the manufacturer's protocol. In the PUI group, irrigants were delivered according to the manufacturer's protocol (NSK Varios 970, NSK, Japan). In the EV group, the irrigant was delivered via the delivery/ evacuation tip at the orifice level.

After irrigation protocols, the teeth were marked at 1 and $3 \mathrm{~mm}$ from the apical foramen with a scalpel. A one-fourth round bur was used to deepen the marks by creating a shallow groove. The teeth were fixed with formalin for $24 \mathrm{~h}$, then decalcified in a specific solution ( $7 \%$ nitric acid-formalin decalcification solution) for 7 days. Using a precise saw, the decalcified roots were cut at the shallow grooves formed at the 1 and $3 \mathrm{~mm}$ level from the apex. Serial sections $4 \mu \mathrm{m}$ in thickness were placed on glass slides, observed by optical microscopy at $\times 128$ magnification, and digitally photographed.

The images were captured using a Leica MZ75 stereomicroscope (Meyer Instruments, Houston, TX, USA) and 
Table 2. Amounts of Remaining Debris at 1 and $3 \mathrm{~mm}$ From the Apex

\begin{tabular}{lll}
\hline Debris (\%) & Mean \pm SD & P value \\
\hline $1 \mathrm{~mm}$ & $10.59 \pm 5.96$ & $0.001^{* *}$ \\
$3 \mathrm{~mm}$ & $6.46 \pm 3.14$ & \\
\hline
\end{tabular}

Student's $t$-test. ${ }^{* *} \mathrm{P}<0.01$.

the Leica QWin software (Meyer Instruments) was used to calculate in pixels the amount of debris remaining in the root canal space. The amount of debris remaining in each root canal was quantified as a percentage of the root canal lumen area. The means were then calculated and compared. Differences in the amounts of remaining debris among the groups were evaluated by one-way analysis of variance (ANOVA) and Student's $t$-tests.

\section{Results}

Amounts of remaining root canal debris in the four experimental groups are shown in Table 1 . The amount of debris remaining at $1 \mathrm{~mm}$ was $18.11 \%$ in the NI group, $7.12 \%$ in the SAF group, $12.71 \%$ in the PUI group and $4.42 \%$ in the EV group. The amount of debris remaining at $3 \mathrm{~mm}$ was $9.61 \%$ in the NI group, $5.22 \%$ in the SAF group, $8.42 \%$ in the PUI group and $2.61 \%$ in the EV group. The amount of debris at $1 \mathrm{~mm}$ in the EV group was significantly lower than in the other groups $(\mathrm{P}<0.01)$. The amount of debris at $1 \mathrm{~mm}$ in the PUI group was significantly higher than in the SAF and EV groups $(\mathrm{P}<0.01)$. The amount of debris at $3 \mathrm{~mm}$ in the $\mathrm{EV}$ group was significantly lower than in the other groups $(\mathrm{P}<$ $0.01)$. The percentages of the remaining root canal debris at 1 and $3 \mathrm{~mm}$ from the apex are shown in Table 2. The amount of debris remaining at $3 \mathrm{~mm}$ was significantly lower than at 1 $\mathrm{mm}(\mathrm{P}<0.01)$. The amount of debris remaining in each root canal lumen area is shown in Figure 1.

\section{Discussion}

Syringe irrigation is a method of irrigant delivery widely accepted among clinicians. This method may be effective in cleaning the coronal third of the root canals but may not clean the apical third because predictable delivery of irrigant to the WL with NI may not be achieved [20, 21]; the irrigant can progress only $1 \mathrm{~mm}$ farther from the needle tip [4]. Lower positive pressure to deliver the irrigating solution

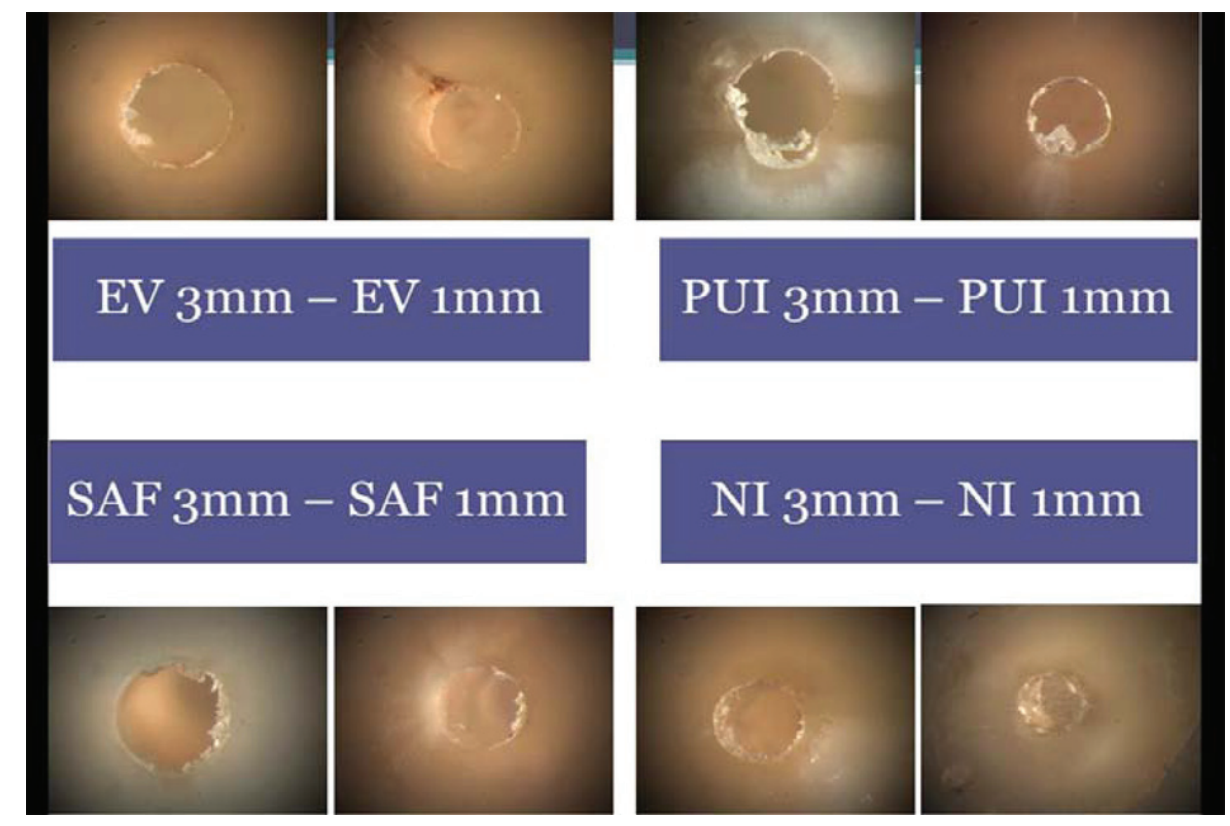

Figure 1. The amount of debris remaining in each root canal lumen area. 
does not allow sufficient irrigant to reach the WL.

However, greater positive pressure and placement of the needle closer to the WL could increase the likelihood of periapical irrigant extrusion. In the clinical setting, the root canal behaves as a closed-end channel because the root is enclosed within the bony socket. This results in gas entrapment at its closed end, producing the vapor-lock effect which prevents the irrigant reaching the WL. This phenomenon can be overcome by using dynamic irrigation systems because they avoid air entrapment due to the continuous supply of fresh irrigant to the WL by negative pressure [22].

The irrigation needle should be placed as close to the apex of the root canal as possible to remove debris effectively [23]. However, the needle tip is placed short of the WL during root canal irrigation to prevent binding to the dentinal walls. Additionally, a conventional irrigating needle cannot be inserted to the WL to prevent irrigant extrusion. For these reasons, the experiment was designed to place the needle a minimum of $2 \mathrm{~mm}$ short of the WL and use an up and down motion. Even in cases in which the needle could reach the WL, the inserted tip was placed at least $2 \mathrm{~mm}$ short of the WL.

In the EV system, the size of the microcannula is equivalent to ISO size 32 and the tip can reach the WL in the root canal enlarged to MAF \#30. The irrigant was supplied from the pulp chamber and removed by application of negative pressure through the macrocannula or microcannula. The manufacturer claimed that their system could minimize extrusion of irrigant. Recently, Desai and Himel (18) demonstrated that the EV system did not extrude root canal irrigants.

In this experiment, the EV system was found to be effective in removing debris at the apical part of the root canal compared with conventional NI, SAF irrigation, and PUI. Nielsen and Craig-Baumgartner [17] reported similar results; however, they used a volume of irrigant that differed depending on the irrigation method. In the present study, an identical volume of irrigant was used regardless of the irrigation method.

In the present study EV exhibited greater efficacy that the other irrigation techniques in the apical $3 \mathrm{~mm}$ of the root canal. Although an identical volume of EDTA was used in both groups, the more efficient smear layer removal by EV was likely due to the turbulence created by the negative pressure in the EV system. Additionally, the direction of fluid flow was from the coronal to apical area in EV, but apical to coronal in conventional NI [24].

Additionally, EV can deliver the irrigating solution to the WL with minimal risk of periapical extrusion. The mechanical flushing action of the irrigant is improved using $\mathrm{EV}$, as the irrigant can be introduced in the root canal under pressure. EV avoids air entrapment due to the continuous supply of fresh irrigant to the WL by negative pressure [25]. Additionally, as the apical size increases, the likelihood of the holes in the microcannula contacting the root canal wall and becoming blocked decreases. The larger area surrounding the microcannula allows an increased volume of irrigant to reach the microcannula tip, resulting in effective smear layer removal. Nielsen and Craig-Baumgartner [17] reported that the performance of EV was superior to that of syringe irrigation in terms of removing debris from the apical third of the root canal.

Thorough cleaning of the root canal depends on effective irrigant delivery, solution agitation [26] and its direct contact with the entire canal wall, particularly in the apical third [26, 27]. SAF uses an irrigation device (Vatea; ReDent-Nova) that provides continuous irrigant flow during use. Because SAF is hollow, the irrigant enters the full length of the canal and is activated by the vibrating motion of the file's metal lattice, reportedly facilitating cleaning and debridement [10]. We used SAF in our study due to its continuous irrigation; however, SAF showed more apical extrusion than the other techniques and considerably more than the EV system. This may be explained by the simultaneous effects of both the $\mathrm{SAF}$ and the continuous flow of the irrigation solution. The Vatea pump, not the clinician, "controls" the irrigation procedure, which may lead to uncontrolled and uncalculated irrigation and consequently, apical extrusion.

PUI is generally accepted as more effective than conventional syringe-and-NI in terms of eliminating pulp tissue and dentin debris. The difference may be due to the ultrasound creating a higher irrigant flow rate in the canal, eliminating more debris, improving access of the chemical product to the accessory canals, and even the flushing effect of ultrasound but not manual irrigation [28]. Our results showed that in addition to these positive attributes, PUI may also push the $\mathrm{NaOCl}$ solution into periapical tissues, similar to other irrigation techniques, but showed significantly more apical extrusion than the EV technique.

In the present study, complete removal of the smear layer was not possible in the apical third of the root canal even using EV, possibly due to the dentinal tubules being irregular and fewer in number. Whittaker and Kneale [29] reported that fewer dentinal tubules per unit area were present in the apical third of the root canal wall. However, Carrigan et al [30] reported that evaluation of the apical third of the root canal for smear layer removal was difficult, possibly due to the smaller size of the dentinal tubules, which are often sclerosed compared with those in the coronal and middle thirds.

\section{Conclusion}

EV irrigation resulted in significantly less debris at both 1 and $3 \mathrm{~mm}$ from the apex compared with the other irrigation techniques. All of the irrigation techniques showed better results at $3 \mathrm{~mm}$ from the apex compared to at $1 \mathrm{~mm}$. However, none of the four techniques completely removed the smear layer from the apical third of the root canal. 


\section{Conflicts of Interest}

The authors deny any conflicts of interest.

\section{References}

1. Cobankara FK, Adanr N, Belli S. Evaluation of the influence of smear layer on the apical and coronal sealing ability of two sealers. J Endod. 2004;30(6):406-409.

2. Goldman M, Goldman LB, Cavaleri R, Bogis J, Lin PS. The efficacy of several endodontic irrigating solutions: a scanning electron microscopic study: Part 2. J Endod. 1982;8(11):487-492.

3. Goldman M, White RR, Moser CR, Tenca JI. A comparison of three methods of cleaning and shaping the root canal in vitro. J Endod. 1988;14(1):7-12.

4. Mayer BE, Peters OA, Barbakow F. Effects of rotary instruments and ultrasonic irrigation on debris and smear layer scores: a scanning electron microscopic study. Int Endod J. 2002;35(7):582-589.

5. Gulabivala K, Patel B, Evans G, Ling Y. Effects of mechanical and chemical procedures on root canal surfaces. Endodont Top. 2005;10:103-122.

6. Moser JB, Heuer MA. Forces and efficacy in endodontic irrigation systems. Oral Surg Oral Med Oral Pathol. 1982;53(4):425-428.

7. Mitchell RP, Yang SE, Baumgartner JC. Comparison of apical extrusion of $\mathrm{NaOCl}$ using the EndoVac or needle irrigation of root canals. J Endod. 2010;36(2):338-341.

8. Metzger Z, Teperovich E, Zary R, Cohen R, Hof R. The self-adjusting file (SAF). Part 1: respecting the root canal anatomy--a new concept of endodontic files and its implementation. J Endod. 2010;36(4):679-690.

9. Hof R, Perevalov V, Eltanani M, Zary R, Metzger Z. The self-adjusting file (SAF). Part 2: mechanical analysis. J Endod. 2010;36(4):691-696.

10. Metzger Z, Teperovich E, Cohen R, Zary R, Paque F, Hulsmann M. The self-adjusting file (SAF). Part 3: removal of debris and smear layer-A scanning electron microscope study. J Endod. 2010;36(4):697-702.

11. De-Deus G, Accorsi-Mendonca T, de Carvalho e Silva L, Leite CA, da Silva D, Moreira EJ. Self-adjusting file cleaning-shaping-irrigation system improves root-filling bond strength. J Endod. 2013;39(2):254-257.

12. De-Deus G, Barino B, Marins J, Magalhaes K, Thuanne E, Kfir A. Self-adjusting file cleaning-shapingirrigation system optimizes the filling of oval-shaped canals with thermoplasticized gutta-percha. J Endod. 2012;38(6):846-849.

13. Plotino G, Pameijer CH, Grande NM, Somma F. Ultrasonics in endodontics: a review of the literature. J Endod. 2007;33(2):81-95.

14. Carver K, Nusstein J, Reader A, Beck M. In vivo an- tibacterial efficacy of ultrasound after hand and rotary instrumentation in human mandibular molars. J Endod. 2007;33(9):1038-1043.

15. van der Sluis LW, Wu MK, Wesselink PR. The evaluation of removal of calcium hydroxide paste from an artificial standardized groove in the apical root canal using different irrigation methodologies. Int Endod J. 2007;40(1):52-57.

16. van der Sluis LW, Shemesh H, Wu MK, Wesselink PR. An evaluation of the influence of passive ultrasonic irrigation on the seal of root canal fillings. Int Endod J. 2007;40(5):356-361.

17. Nielsen BA, Craig Baumgartner J. Comparison of the EndoVac system to needle irrigation of root canals. J Endod. 2007;33(5):611-615.

18. Desai P, Himel V. Comparative safety of various intracanal irrigation systems. J Endod. 2009;35(4):545-549.

19. Fukumoto Y, Kikuchi I, Yoshioka T, Kobayashi C, Suda H. An ex vivo evaluation of a new root canal irrigation technique with intracanal aspiration. Int Endod J. 2006;39(2):93-99.

20. Siqueira JF, Jr., Araujo MC, Garcia PF, Fraga RC, Dantas CJ. Histological evaluation of the effectiveness of five instrumentation techniques for cleaning the apical third of root canals. J Endod. 1997;23(8):499-502.

21. Chow TW. Mechanical effectiveness of root canal irrigation. J Endod. 1983;9(11):475-479.

22. Tay FR, Gu LS, Schoeffel GJ, Wimmer C, Susin L, Zhang K, Arun SN, et al. Effect of vapor lock on root canal debridement by using a side-vented needle for positive-pressure irrigant delivery. J Endod. 2010;36(4):745750.

23. Abou-Rass M, Piccinino MV. The effectiveness of four clinical irrigation methods on the removal of root canal debris. Oral Surg Oral Med Oral Pathol. 1982;54(3):323328.

24. Abarajithan M, Dham S, Velmurugan N, Valerian-Albuquerque D, Ballal S, Senthilkumar H. Comparison of Endovac irrigation system with conventional irrigation for removal of intracanal smear layer: an in vitro study. Oral Surg Oral Med Oral Pathol Oral Radiol Endod. 2011;112(3):407-411.

25. Shin SJ, Kim HK, Jung IY, Lee CY, Lee SJ, Kim E. Comparison of the cleaning efficacy of a new apical negative pressure irrigating system with conventional irrigation needles in the root canals. Oral Surg Oral Med Oral Pathol Oral Radiol Endod. 2010;109(3):479-484.

26. Gu LS, Kim JR, Ling J, Choi KK, Pashley DH, Tay FR. Review of contemporary irrigant agitation techniques and devices. J Endod. 2009;35(6):791-804.

27. Zehnder M. Root canal irrigants. J Endod. 2006;32(5):389-398.

28. van der Sluis LW, Wu MK, Wesselink PR. A comparison between a smooth wire and a K-file in removing 
artificially placed dentine debris from root canals in resin blocks during ultrasonic irrigation. Int Endod J. 2005;38(9):593-596.

29. Whittaker DK, Kneale MJ. The dentine-predentine interface in human teeth. A scanning electron microscope study. Br Dent J. 1979;146(2):43-46.

30. Carrigan PJ, Morse DR, Furst ML, Sinai IH. A scanning electron microscopic evaluation of human dentinal tubules according to age and location. J Endod. 1984;10(8):359-363. 